\title{
Outcome of Pregnancy in the Era of Pegylated Interferon Alpha 2a in Females with Essential Thrombocythemia: An Experience from Qatar
}

\author{
Mohammad Abu-Tineh ${ }^{a} \quad$ Nancy Kassem ${ }^{b}$ \\ Mohammad Abdul-Jaber Abdullac Omar Mohammad Ismail ${ }^{c}$ \\ Rola Ghasoub ${ }^{b}$ Mahmood B. Aldapt ${ }^{c}$ Mohamed A. Yassin ${ }^{c}$ \\ aDepartment of Medical Education, Hamad Medical Corporation (HMC), Doha, Qatar; \\ bNational Center for Cancer Care and Research, Department of Pharmacy, HMC, Doha, \\ Qatar; 'National Center for Cancer Care and Research, Department of Oncology - \\ Hematology and BMT Section -, HMC, Doha, Qatar
}

\section{Keywords}

Essential thrombocythemia · Myeloproliferative neoplasms · Interferon · PEG-IFN alpha-2A · Pregnancy

\section{Abstract}

Myeloproliferative neoplasms are a diversified group of diseases of the hematopoietic stem cell, such as essential thrombocythemia (ET) and polycythemia vera. They are mainly caused by mutations in the following genes: JAK2, CALR, and MPL. All carry an increased risk to transform into acute leukemia or chronic myelogenous leukemia along with thrombosis and hemorrhagic complications. Treatment of such disorders during pregnancy is a challenging footstep, given the high risk of complications for both the mother and the fetus. Here, we report about two pregnant females with ET that has been treated with pegylated interferon alpha with safe and effective outcome.

(c) 2020 The Author(s).

Published by S. Karger AG, Basel

\section{Introduction}

Myeloproliferative neoplasms (MPNs), including essential thrombocythemia (ET) and polycythemia vera, are commonly diagnosed in the sixth decade, but as many as $20 \%$ of all patients are younger than 40 years [1]. MPN is caused by the clonal proliferation of myeloid

Mohammad Abu-Tineh, Nancy Kassem, and Mohamed A. Yassin contributed equally to this work. 
cells, which results in elevated numbers of circulating platelets, thrombotic or hemorrhagic episodes, and occasional leukemic transformation [2]. Familial ET, or inherited thrombocythemia or thrombocytosis, is a rare disorder, which is autosomal dominant, usually presenting as sporadic ET. But studies have shown that it is likely caused by a genetic predisposition to acquire somatic mutations, rather than to direct inheritance of germline mutations. Around $90 \%$ of all cases have a somatically acquired driver mutation in the genes JAK2, CALR, or MPL. This is causing an upregulation of the JAK-STAT target genes, demonstrating the central importance of this pathway in the pathogenesis of ET [3].

The incidence of the disease is controlled by multiple factors, such as race, sex, and age. There is a female preponderance with an approximate female-to-male ratio of 2:1 [4]. Around half of the patients with ET are detected incidentally upon doing blood investigations for other reasons. Patients with ET show a higher incidence of thrombosis and hemorrhage; thrombosis as in, e.g., cerebrovascular accident, myocardial infarction, superficial thrombophlebitis, deep vein thrombosis, or pulmonary embolism, mainly due to qualitative and quantitative platelet alterations [5]. Treatment is usually aimed to prevent hemorrhage or thrombosis occurrence and their complications.

The treatment of ET during pregnancy is a challenging decision, as it holds the risk of abortion, thrombotic complications, and other risks. Today, there is limited data on the use of pegylated interferon alpha (PEG-IFN $\alpha$ ), with regard to treatment and outcome on the basis of disease control and safety.

\section{Cases Presentations}

\section{Case 1}

A 34-year-old American female was diagnosed with ET according to the 2008 WHO criteria and started on PEG-IFN $\alpha 50 \mu \mathrm{g}$ for 2 weeks; then, the dose was escalated to $135 \mu \mathrm{g}$ subcutaneous once weekly. The patient and her husband were offered conventional interferon as a safe alternative, but they insisted on continuing on PEG-IFN $\alpha$. The patient was referred to the high-risk pregnancy unit of the maternity hospital for a close follow-up. Eventually, she gave birth to a healthy baby girl.

Case 2

A 31-year-old Egyptian female was diagnosed with ET according to the 2008 WHO criteria and started on PEG-IFN $\alpha 50 \mu \mathrm{g}$ for 2 weeks; then, the dose was escalated to $135 \mu \mathrm{g}$ subcutaneous once weekly. This patient became pregnant while on PEG-IFN $\alpha$. The patient and her husband were offered conventional interferon as a safe alternative, but they insist on continuing on PEG-IFN $\alpha$. The patient was referred to the high-risk pregnancy unit of the maternity hospital for a close follow-up. Eventually, she gave birth to a healthy baby boy.

\section{Discussion}

Pregnancy is not contraindicated in patients with ET, as a normal pregnancy with normal fetal outcome is possible in patients with ET, even without therapy. However, ET in pregnant women may lead to multiple problems for the mother and/or the fetus [6]. Therefore, it is advisable to discuss possible complications associated with the current treatment options and reach a decision with the parents-to-be preferably before conception. In high-risk women with ET who are either pregnant or planning to become pregnant, therapy with a platelet- 
lowering agent is essential to prevent recurrent thrombosis. Current treatment options include aspirin, IFN $\alpha$, and thrombapheresis.

Aspirin works by inhibiting the cyclooxygenase activity of the platelets causing a reduction in platelet release and aggregation, which in return decreases the chances of thromboembolic complications. An advantage of aspirin therapy is its associated reduction of preeclampsia in patients at high risk for this complication. On the other hand, aspirin has also been well associated with complications during pregnancy, especially intracranial hemorrhage during delivery [6]. Thrombapheresis is a safe therapy, especially in acute situations such as massive thrombosis or hemorrhage. It can be applied during pregnancy without any risk for the fetus; however, disadvantages of this therapy are that apheresis is expensive, time-consuming, and has to be repeated frequently [6].

Several studies have shown the effectiveness of controlling platelet counts and inducing molecular responses in ET [7-14]; in addition, IFN $\alpha$ is not considered leukemogenic nor teratogenic. Several successful pregnancies have been reported in ET patients treated with IFN $\alpha$ [15-22]; however, due to its significant side effects and inconvenient frequency of administration, its use is limited to high-risk pregnant women or to patients where treatment with hydroxyurea failed.

PEG-IFN $\alpha$ is a pegylated form of IFN $\alpha$ that is naturally occurring as a biologic response modifier. It has anti-angiogenic, antiproliferative, pro-apoptotic, immunomodulatory, and differentiating properties [12]. PEG-IFN $\alpha$ has a longer half-life and a reduced clearance compared to IFN $\alpha$ and can therefore be given once weekly. Its efficacy has been investigated in several phase 2 trials which showed efficacy in controlling platelet counts in patients with ET [23-25]. However, only sparse data have been published on the safety of its use in pregnant women. A recent observational study [26], on 10 pregnancies in women with ET treated with PEG-IFN $\alpha$, suggested efficacy and safety of PEG-IFN $\alpha$ in pregnant women with ET. In this study, the median dose of PEG-IFN $\alpha$ was $270 \mu \mathrm{g} / \mathrm{month}$ (range 90-1,080 $\mu \mathrm{g} / \mathrm{month}$ ), while in our reported cases, we used a fixed dose of $135 \mu \mathrm{g} /$ week $(540 \mu \mathrm{g} / \mathrm{month})$. Moreover, in Beauverd et al.'s study [26], aspirin and/or low-molecular-weight heparin was used in all cases in addition to PEG-IFN $\alpha$.

Here, we presented 2 cases of pregnant women with ET who opted to take PEG-INF $\alpha$ during their pregnancy. Both women had normal deliveries of healthy babies with normal birth weight and cognitive functions. The metabolic screenings and thyroid function tests returned normal for the two babies.

\section{Conclusion}

PEG-INF $\alpha$ might be a promising upcoming treatment for ET during pregnancy, given our report of two cases and their encouraging outcome. However, the efficacy and safety of PEG-IFN $\alpha$ for MPN, and mainly for ET treatment, have been investigated only in one recent small observational study. We would like to highlight the importance of further studies of such possible treatment for the management of MPN during pregnancy.

\section{Acknowledgment}

We thank Qatar National Library for funding this article, and we thank the internal medicine residency program at Hamad Medical Corporation. 
Abu-Tineh et al.: Outcome of Pregnancy in the Era of Pegylated Interferon Alpha 2a

\section{Statement of Ethics}

This case report was approved by the Hamad Corporation Medical Research Center.

\section{Disclosure Statement}

The authors have nothing to disclose.

\section{Funding Sources}

This article was funded by Qatar National Library.

\section{Author Contributions}

Mohammad Abu-Tineh and Nancy Kassem: Writing of the Manuscript. Mohammad Abdul-Jaber Abdulla, Omar Mohammad Ismail, Mahmood B. Aldapt, Rola Ghasoub: Clinical Management. Mohamed A. Yassin: Writing and Editing.

\section{References}

1 How CJ, Leiva O, Bogue T, Fell G, Bustoros M, Connell NT, et al. Pregnancy outcomes, risk factors, and gestational cell count trends in pregnant women with essential thrombocythemia and polycythemia vera. Blood. 2019;134(Suppl 1):4172.

2 Yassin MA, Al-Dewik NI, ElAyoubi H, Cassinat B. Familial essential thrombocythemia among Qatari tribes. Blood. 2013;122(21):5244.

3 Al-Dewik N, Ben-Omran T, Zayed H, Trujillano D, Kishore S, Rolfs A, et al. Clinical exome sequencing unravels new disease-causing mutations in the myeloproliferative neoplasms: a pilot study in patients from the state of Qatar. Gene. 2019;689:34-42.

4 Yassin AM, Taher A, Mathews V, Hou HA, Shamsi T, et al. Myeloproliferative neoplasms in Asia, including Middle East, Turkey, and Algeria: epidemiological indices and treatment practice patterns from the Multinational, Multicenter, Observational MERGE Registry. Blood. 2018;132(1):5461.

5 Schafer AI. Molecular basis of the diagnosis and treatment of polycythemia vera and essential thrombocythemia. Blood. 2006 Jun 1;107(11):4214-22.

6 Vantroyen B, Vanstraelen D. Management of essential thrombocythemia during pregnancy with aspirin, interferon alpha-2a and no treatment. A comparative analysis of the literature. Acta Haematol. 2002;107(3):15869.

7 Lengfelder E, Griesshammer M, Hehlmann R. Interferon-alpha in the treatment of essential thrombocythemia. Leuk Lymphoma. 1996 Jan 1;22(Suppl 1):135-42.

8 Elliott MA, Tefferi A. Interferon- $\alpha$ therapy in polycythemia vera and essential thrombocythemia. Semin Thromb Hemost. 1997 Oct;23(5):463-72.

9 Gilbert HS. Long term treatment of myeloproliferative disease with interferon- $\alpha-2 b$ : feasibility and efficacy. Cancer. 1998 Sep 15;83(6):1205-13.

10 Saba R, Jabbour E, Giles F, Cortes J, Talpaz M, O’Brien S, et al. Interferon $\alpha$ therapy for patients with essential thrombocythemia: final results of a phase II study initiated in 1986. Cancer. 2005 Jun 15;103(12):2551-7.

11 Kiladjian JJ, Chomienne C, Fenaux P. Interferon- $\alpha$ therapy in bcr-abl-negative myeloproliferative neoplasms. Leukemia. 2008 Nov;22(11):1990-8.

12 Kiladjian JJ, Mesa RA, Hoffman R. The renaissance of interferon therapy for the treatment of myeloid malignancies. Blood. 2011 May 5;117(18):4706-15.

13 Quintás-Cardama A, Kantarjian H, Manshouri T, Luthra R, Estrov Z, Pierce S, et al. Pegylated interferon alfa-2a yields high rates of hematologic and molecular response in patients with advanced essential thrombocythemia and polycythemia vera. J Clin Oncol. 2009 Nov 10;27(32):5418. 
14 Verger E, Cassinat B, Chauveau A, Dosquet C, Giraudier S, Schlageter MH, et al. Clinical and molecular response to interferon- $\alpha$ therapy in essential thrombocythemia patients with CALR mutations. Blood. 2015;126(24): 2585-91.

15 Harrison C. Pregnancy and its management in the Philadelphia negative myeloproliferative diseases. $\mathrm{Br} J$ Haematol. 2005 May;129(3):293-306.

16 Delage R, Demers C, Cantin G, Roy J. Treatment of essential thrombocythemia during pregnancy with interferon- $\alpha$. Obstet Gynecol. 1996 Jan 1;87(5):814-7.

17 Milano V, Gabrielli S, Rizzo N, Vianelli N, Cugliotta L, Orsini LF, et al. Successful treatment of essential thrombocythemia in a pregnancy with recombinant interferon- $\alpha 2 a$. J Maternal-Fetal Med. 1996 Jan 1;5(2):74-8.

18 Martinelli P, Martinelli V, Agangi A, Maruotti GM, Paladini D, Ciancia R, et al. Interferon alfa treatment for pregnant women affected by essential thrombocythemia: case reports and a review. Am J Obstet Gynecol. 2004 Dec 1;191(6):2016-20.

19 Griesshammer M, Heimpel H, Pearson TC. Essential thrombocythemia and pregnancy. Leuk Lymphoma. 1996 Jan 1;22(Suppl 1):57-63.

20 Eliyahu S, Shalev E. Essential thrombocythemia during pregnancy. Obstet Gynecol Surv. 1997 Apr 1;52(4): 243-7.

21 Yassin MA, Al-Dewik N, El-Ayoubi HR. Pegylated interferon-alfa-2a induces complete hematologic and molecular responses with low toxicity in essential thrombocythemia. Blood. 2012;1734..

22 Yassin MA, Al-Dewik NI, ElAyoubi H, Cassinat B. Efficacy and safety of pegelated interferon alpha2a once monthly compared to once weekly dose in patients with essential thrombocythemia. Blood. 2013;122(21): 4054.

23 Samuelsson J, Hasselbalch H, Bruserud O, Temerinac S, Brandberg Y, Merup M, et al.; Nordic Study Group for Myeloproliferative Disorders. A phase II trial of pegylated interferon $\alpha-2 b$ therapy for polycythemia vera and essential thrombocythemia: feasibility, clinical and biologic effects, and impact on quality of life. Cancer. 2006 Jun 1;106(11):2397-405.

24 Langer C, Lengfelder E, Thiele J, Kvasnicka HM, Pahl HL, Beneke H, et al. Pegylated interferon for the treatment of high risk essential thrombocythemia: results of a phase II study. Haematologica. 2005 Jan 1;90(10):1333-8.

25 Gowin K, Thapaliya P, Samuelson J, Harrison C, Radia D, Andreasson B, et al. Experience with pegylated interferon $\alpha$-2a in advanced myeloproliferative neoplasms in an international cohort of 118 patients. Haematologica. 2012 Oct 1;97(10):1570-3.

26 Beauverd Y, Radia D, Cargo C, Knapper S, Drummond M, Pillai A, et al. Pegylated interferon alpha-2a for essential thrombocythemia during pregnancy: outcome and safety. A case series. Haematologica. 2016 May; 101(5):e182. 